\title{
Weighting Under Ambiguous Preferences and Imprecise Differences in a Cardinal Rank Ordering Process
}

\author{
Mats Danielson \\ Dept. of Computer and Systems Sciences, Stockholm University \\ Forum 100, SE-164 40 Kista, Sweden \\ E-mail:mad@dsv.su.se \\ Love Ekenberg \\ Dept. of Computer and Systems Sciences, Stockholm University \\ Forum 100, SE-164 40 Kista, Sweden \\ E-mail:lovek@dsv.su.se \\ Aron Larsson \\ Dept. of Computer and Systems Sciences, Stockholm University \\ Forum 100, SE-164 40 Kista, Sweden \\ E-mail: aron@dsv.su.se \\ Mona Riabacke \\ Dept. of Computer and Systems Sciences, Stockholm University \\ Forum 100, SE-164 40 Kista, Sweden \\ E-mail:mona.riabacke@gmail.com \\ www.dsv.su.se \\ Received 16 December 2012 \\ Accepted 11 July 2013
}

\begin{abstract}
The limited amount of good tools for supporting elicitation of preference information in multi-criteria decision analysis (MCDA) causes practical problem. In our experiences, this can be remedied by allowing more relaxed input statements from decision-makers, causing the elicitation process to be less cognitively demanding. Furthermore, it should not be too time consuming and must be able to actually use of the information the decision-maker is able to supply. In this paper, we propose a useful weight elicitation method for MAVT/MAUT decision making, which builds on the ideas of rank-order methods, but increases the precision by adding numerically imprecise cardinal information as well.
\end{abstract}

Keywords: Multi-criteria decision analysis, imprecision, criteria weights, elicitation.

\section{Introduction}

During the last few decades, the field of decision analysis has developed as a structured approach to formally analysing decision problems. The field is based on research from several disciplines, in particular organisation theory, business administration, psychology, statistics, computer science, and philosophy. The main idea of decision analysis is to provide means and methods for helping people make better decisions ${ }^{2}$. Over the years, research on the formal properties of decision making has moved from the initial studies of a rational theory of choice based upon single objective decision problems towards pursuing the design of decision support methods for more realistic decision making situations with multiple objectives - Multi-Criteria Decision Analysis (MCDA). After identifying objectives and the available courses of action, the possible consequences 
are analysed formally on the basis of the provided input data.

Multi-Attribute Value Theory, MAVT, and MultiAttribute Utility Theory, MAUT ${ }^{1,3,4}$, are the most widely used MCDA methods, but there are others, such as the Analytic Hierarchy Process ${ }^{5}$ (AHP), the ELECTRE family of methods (cf., e.g., Ref. 6), and PROMETHEE (cf., e.g., Ref. 7).

During the last half century, decision theory has developed significantly in a multitude of ways, but decision analysis tools are still seldom utilized to aid decision making processes in most organizations ${ }^{8}$, and people rarely perform formal analysis to complex problems ${ }^{2}$. Behavioural concerns ${ }^{9}$ have not, despite a quite substantial activity within descriptive theories, got sufficient attention and there is still a lack of support for the decision analytic process itself ${ }^{10}$.

There are a number of models for how decision processes should be to be rational where a number of components must be obtained throughout the process. For example, in Ref. 11 a standard model of a decision analysis process is described, where the elicitation is one of the crucial modelling parts. However, the main focus within the research field has been on other components.

In this paper we focus on the elicitation part of the decision analysis process, i.e. the interactive part of the process where decision-makers express their preferences and priorities. Many papers have discussed problematic elements of the elicitation process in decision analysis applications, such as cognitive demand (cf., e.g., Ref. 12 and Ref. 4), difficulties in judging and expressing precise input, (cf., e.g., Refs. 13-14), and biases and inconsistencies that arise (cf., e.g., Refs. 1516). A systematic mistake in applied MAVT/MAUT decision making is the absence of adequate elicitation methods when searching for a decision-makers attitude to the relative importance of each criterion ${ }^{17}$. Furthermore, providing precise input is usually too demanding and there is a clear need for other elicitation methods (cf., e.g., Refs. 18-19)

In this paper, we discuss the elicitation of MAVT/ MAUT criteria weights using a cardinal interpretation of initially ordinal or numerically imprecise cardinal decision-maker statements, where imprecision is modelled by means of linear constraints derived from a onedimensional graphical representation of numerically imprecise criteria weight rankings. A process conform- ing to SMART/SWING weighting is proposed, relaxing the need to make ratio statements between utility differences.

\subsection{Relative Importance of Criteria}

In MAUT, the most common form of value function used is the additive model which builds on the utility principle. Given an alternative $A_{i}$, let $C_{i}$ be a (finite) set of possible but uncertain consequences associated with some performance value and some criterion $\mathrm{G}_{s}$. Then the expected utility under criterion $\mathrm{G}_{s}, U_{s}\left(A_{i}\right)$, is given by

$$
U_{s}\left(A_{i}\right)=\sum_{c_{j k} \in C_{i}} p_{j k} u\left(c_{j k}\right)
$$

where $p_{j k}$ is the probability of the $k$ :th consequence in $C_{i}$, and $u\left(c_{j k}\right)$ is the utility value of the $k$ :th consequence's performance value $c_{j k}$. Utilities are defined on an interval scale and a common practice is to let $u(\cdot)$ assume values in the closed interval $[0,1]$ such that the worst consequence (least preferred) maps to zero and the best consequence (most preferred) maps to one. In the presence of more than one criterion, the overall value of alternative $A_{i}$, here denoted $V\left(A_{i}\right)$, is given by

$$
V\left(A_{i}\right)=\sum_{j=1}^{n} w_{j} U_{j}\left(A_{i}\right)
$$

where is $U_{j}\left(A_{i}\right)$ is the expected utility of the alternative relative to criterion $G_{j}$. Further, $w_{j}$ is the weight assigned to this criterion. A common requirement here is that the weights are normalised, i.e. $\sum w_{i}=1$.

Note that the interpretation of these weights in the function $V()$ is dependent on the decision method and its accompanied weight elicitation method used ${ }^{20}$. From a decision-maker's point-of-view, however, the weights are generally supposed to be a numeric value representing a common "relative importance of each criterion". One suggestion of a formal interpretation of weights equipped with usable elicitation questions for a decision-maker in the elicitation is the SMART/SWING process ${ }^{3}$.

To illustrate this, given a decision problem with $m$ alternatives in a set $\mathbf{A}=\left\{A_{1}, A_{2}, \ldots, A_{m}\right\}$ of alternatives and $n$ criteria in a set $\mathbf{G}=\left\{G_{1}, G_{2}, \ldots, G_{n}\right\}$, let ${ }^{i} c_{\uparrow}$ be the consequence having the best performance relative to 
criterion $G_{i}$ and let ${ }^{i} c_{\downarrow}$ be the consequence having the worst performance relative to criterion $G_{i}$. Then the function $u_{i}(c)$ is defined such that $u_{i}\left({ }^{i} C_{\downarrow}\right)=0$ and $u_{i}\left({ }^{i} C_{\uparrow}\right)=$ 1. If $u_{i}(\cdot)$ also is linear, i.e.

$$
u_{i}(c)=\frac{\left|c-{ }^{i} c_{\downarrow}\right|}{\left|{ }^{i} c_{\uparrow}-{ }^{i} c_{\downarrow}\right|}
$$

then this is the widely employed idea of "proportional scores". The relative importance of the criteria is thus expressed by the magnitude of the weights $w_{i}$ such that if $w_{i}>w_{j}$ then $G_{i}$ is more important than $G_{j}$ and if $w_{i}=$ $w_{j}$ the two criteria are considered equally important for a decision-maker. From this, it now follows that

$$
\begin{aligned}
& w_{i}= \\
& w_{1} u_{1}\left({ }^{1} c_{\downarrow}\right)+\ldots+w_{i-1} u_{i-1}\left({ }^{i-1} c_{\downarrow}\right)+ \\
& w_{i} u_{i}\left({ }^{i} c_{\uparrow}\right)+w_{i+1} u_{i+1}\left({ }^{i+1} c_{\downarrow}\right)+\ldots+w_{n} u_{n}\left({ }^{n} c_{\downarrow}\right)= \\
& w_{1} \cdot 0+\ldots+w_{i-1} \cdot 0+w_{i} \cdot 1+w_{i+1} \cdot 0+\ldots+w_{n} \cdot 0
\end{aligned}
$$

meaning that the weight for each criterion reflects the desirability of the most preferred consequence for that criterion. The weight ratio $w_{i} / w_{j}$ thus scales the ratio $\left.\left[u_{i}\left({ }^{i} C_{\uparrow}\right)-u_{i}\left({ }^{i} c_{\downarrow}\right)\right] /\left[u_{j}{ }^{j} c_{\uparrow}\right)-u_{j}\left({ }^{j} c_{\downarrow}\right)\right]$ to a desired level on behalf of the decision-maker. In other words, the range of possible consequences for each criterion from a preference perspective is implicitly modelled in the weights.

Relying solely on the formal interpretation of weights and promoting it to decision-makers subject to weight elicitation is not a very practical approach nor beneficial for the decision analysis field since it is likely to put too high cognitive demands on the decisionmakers. The concern for the development of weight elicitation processes is to balance the demands put on the decision-makers and the equitability of the weights elicited, i.e. so that they represent the preferences of the decision-makers. In order to represent the preferences, a SMART/SWING weight elicitation process introduces a set of hypothetical decision alternatives $H_{0}$ to $H_{n}$ of the form

$$
\begin{aligned}
& H_{0}:{ }^{1} c_{\downarrow},{ }^{2} c_{\downarrow},{ }^{3} c_{\downarrow},{ }^{4} c_{\downarrow},{ }^{5} c_{\downarrow}, \ldots,{ }^{n} c_{\downarrow} \\
& H_{1}:{ }^{1} C_{\uparrow},{ }^{2} c_{\downarrow},{ }^{3} c_{\downarrow},{ }^{4} C_{\downarrow},{ }^{5} c_{\downarrow}, \ldots,{ }^{n} c_{\downarrow} \\
& H_{2}:{ }^{1} c_{\downarrow},{ }^{2} c_{\uparrow},{ }^{3} c_{\downarrow},{ }^{4} c_{\downarrow},{ }^{5} c_{\downarrow}, \ldots,{ }^{n} c_{\downarrow} \\
& H_{3}:{ }^{1} C_{\downarrow},{ }^{2} c_{\downarrow},{ }^{3} C_{\uparrow},{ }^{4} C_{\downarrow},{ }^{5} c_{\downarrow}, \ldots,{ }^{n} C_{\downarrow} \\
& H_{n}:{ }^{1} c_{\downarrow},{ }^{2} c_{\downarrow},{ }^{3} c_{\downarrow},{ }^{4} c_{\downarrow},{ }^{5} c_{\downarrow}, \ldots,{ }^{n} c_{\uparrow}
\end{aligned}
$$

and the decision-maker is asked to express the differences between each $V\left(H_{i}\right)$ and $V\left(H_{0}\right)$. The hypothetical alternative $H_{j}$ for which this difference is considered to be the largest indicates that $w_{j}$ is the largest of the weights in the system and $H_{j}$ is awarded with 100 points. The remaining $H_{i}$ are then awarded with points between 0 and 100 by the decision-maker indicating the ratio $w_{j} / w_{i}$. The term "swing” originates from the fact that each criterion is swung from worst to best when comparing $H_{0}$ with one of the other hypothetical alternatives. In this manner, the weights reflect relative importance of criteria in the decision context at hand, where the range of possible consequences for each criterion is taken into account.

\subsection{Numerically Imprecise Information}

The implicit assumption in the process described above is that people are really able to assess the values required for analysis accurately, such as the ratio statements mentioned above. Naturally, many concepts, such as knowledge, beliefs, and preferences, are not naturally represented with numeric precision (cf., e.g., Refs. 1314), and the difficulty of eliciting precise decision parameters (utility values, criteria weights, and probabilities on uncertain consequences) have been widely discussed $^{21}$. There are severe inconsistencies with the predictions of the rational model (cf., e.g., Refs. 22 and 15) since many fundamental assumptions regarding elicitations do not hold (cf., e.g., Refs. 23-26).

There is, consequently, much uncertainty present in elicitation, and avoiding precise importance judgments is obviously advantageous for joining theory and practice $^{27,28}$. Ratio weight procedures are difficult to make accurate due to response errors ${ }^{29}$ and numerically imprecise weights have been proposed instead, in particular preferential uncertainties and incomplete information handled in a more cardinally oriented way using convex sets of possible values within intervals ${ }^{30}$. Differences in preferences and judgments can then be represented by value intervals ${ }^{31}$

In the GMAA system (see Ref. 31) the extraction is based on trade-offs among the attributes. The decisionmaker is asked to provide an interval where he or she is indifferent with respect to a lottery and a sure consequence. Another is direct assignment where the decision-maker directly assigns a weight interval to the respective criteria. In Ref. 32, an Interval SMART / SWING method is proposed, allowing interval judgments to represent imprecision during extraction. The extracted weight information is represented by con- 
straints for the attributes' weight ratios, determining feasible region of the weights.

Another class of methods is based on surrogate weights derived from ordinal information. Decisionmakers rank the different criteria. Thereafter the ordering is translated into surrogate (cardinal) weights consistent with the rankings. The conversion is made from ordinal to cardinal weights for use in an additive utility function. There are many proposals on how to convert the rankings provided by the decision-maker into numerical weights, e.g., rank sum weights, rank reciprocal weights $^{33}$, and centroid (ROC) weights ${ }^{12}$. Of the conversion methods suggested, ROC has gained the most recognition $^{34}$. For instance, SMARTER is a decision method having a component to elicit the ordinal information on importance that are then converted to ROC weights. The information loss here is substantial. In many situations, people can with confidence state that some differences in importance are greater than others ${ }^{29}$, which is ignored in pure ordinal approaches. Further, studies have revealed that even the ranking may be subject to ambiguity ${ }^{35}$.

Ordinal ranking of the criteria is effort-saving, but the assumption of knowing the ranking with certainty is indeed strong. There is also a potential information loss in using ordinal rankings only. Furthermore, the number of judgments necessary in order to obtain a sufficient weight system should preferably be reduced. The idea of cardinal rank ordering of weights addresses these concerns.

\section{Cardinal Rank Ordering of Criteria}

The elicitation method described below, Cardinal Rank Ordering of Criteria (CROC), takes both ordinal information and numerically imprecise cardinal relation information into account. In its representational and interpretational aspects, CROC extends rank-order methods such as ROC into a model for ordinal ranking with the possibility of also handling numerically imprecise cardinal information.

\subsection{The CROC process}

In the CROC extraction stage, the decision-maker's criteria ranking is elicited in a three step process; each step providing additional preference information with respect to criteria weights. Thereafter, the information is represented and interpreted. We will henceforth denote the set of $N$ criteria $\mathbf{G}=\left\{G_{1}, \ldots, G_{N}\right\}$ where each criteri- on in the set is associated with a value $x_{i}$ and a criterion weight variable $w_{i}$.

\section{The first step (initial ordering step)}

In the first step, only ordinal information is collected in a criteria ranking. The ranking is done in SWING weighting style supported by a consequence ordering algorithm producing a Hasse diagram of all consequences for each criterion.

Example: Given a criterion $G_{i}$ with the following input statements with respect to the set of five possible consequences

$$
\begin{aligned}
& u_{i}\left({ }^{i} c_{5}\right)>u_{i}\left({ }^{i} c_{3}\right) \\
& u_{i}\left({ }^{i} c_{3}\right)>u_{i}\left({ }^{i} c_{4}\right) \\
& u_{i}\left({ }^{i} c_{4}\right)>u_{i}\left({ }^{i} c_{1}\right) \\
& u_{i}\left({ }^{i} c_{4}\right)>u_{i}\left({ }^{i} c_{2}\right)
\end{aligned}
$$

then the Hasse diagram (Fig. 1) over the set of five consequences is

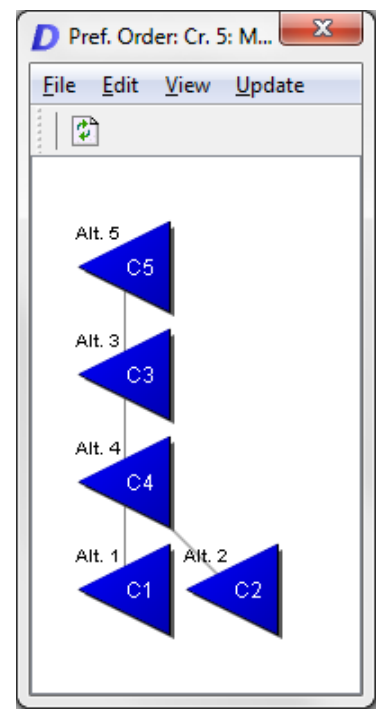

Fig. 1. Consequence order Hasse diagram

The diagram in Fig. 1 above can then be used to support ordinal SWING weighting by means of comparing the differences between the worst and best consequences for each criterion, e.g., the "swing step" between $C_{5}$ and $C_{1}$ or $C_{2}$ and how large this step is compared to the corresponding swing step for others is assessed on an ordinal scale. 


\section{The second step (initial cardinal step)}

Then the decision-maker is assumed to assess the maximum difference $D$ between the weights for the most and the least important criteria. ${ }^{*}$ The highest (lowest) value asserted by the user is denoted by $x_{H}\left(x_{L}\right)$. The anchor points are that $x_{H}$ initially is set to 100 and $x_{L}$. to 0 . A user can thus express the difference between the most and least important criteria by setting $D=x_{H}-x_{L}$ within the open interval $(0,100)$.

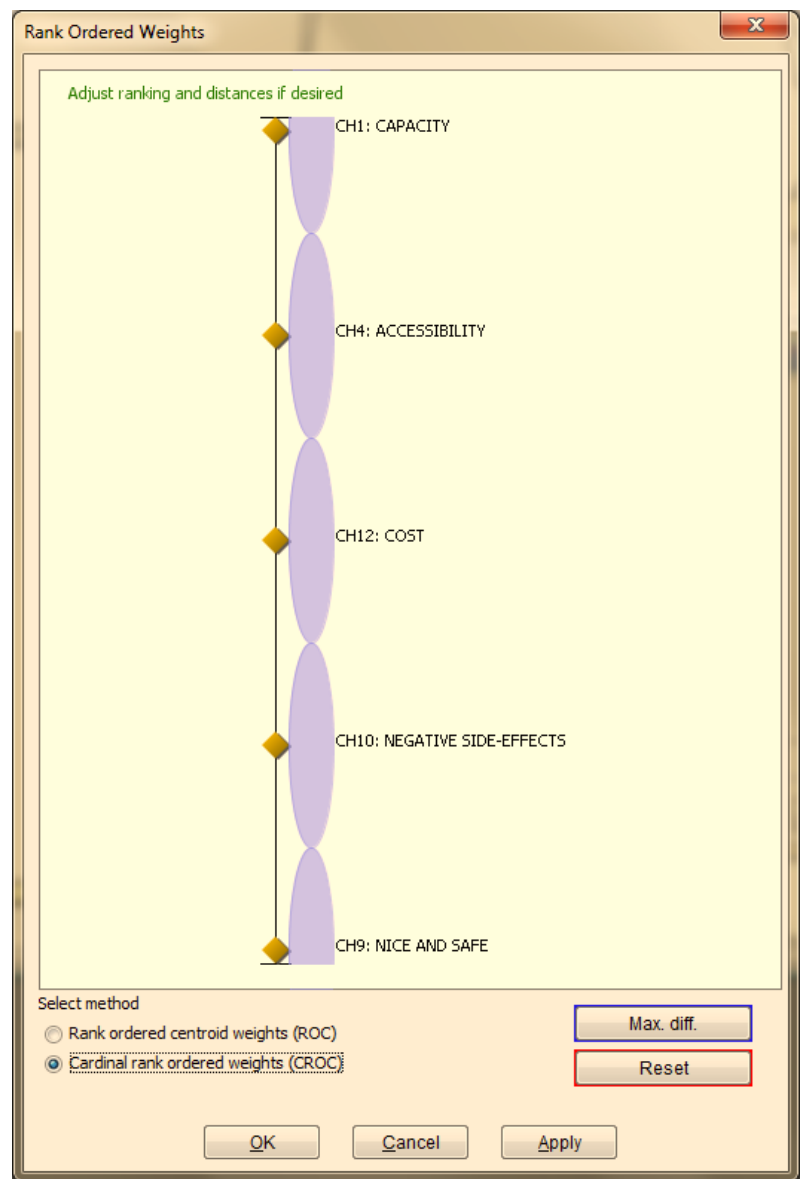

Fig. 2. CROC weight elicitation after the initial ordering step

\section{Third step (cardinal rank ordering step)}

In the cardinal rank ordering step, the criteria are distributed equally, since there is no further information beyond a ranking. The decision-maker is then allowed to adjust the distances and expressing numerically imprecise cardinal importance. Each criterion on the slider corresponds to a region with length equal to the default distance (see Fig. 2). Two criteria overlap when they are

\footnotetext{
* We will below assume that $G_{1}$ is more important than $G_{2}$ which is more important than $G_{3}$ and so forth.
}

closer together than the default distance. If they are further apart compared to the default distance, there is a minimum difference between the criteria. The input to the method thus consists of $x_{L}$ and distances of these kinds. $^{\dagger}$

We then obtain strength information for the differences between the criteria that could more adequately represent the weights. For instance, in Figure 2 initially five criteria in a traffic planning decision situation are being ranked. In the figure, "Capacity" is more important than "Accessibility”, which in turn is more important than "Cost", and so on.

This is the view as the second step commences, where the maximum difference between "Capacity" and "Nice and safe" is asked for. Then numerically imprecise cardinal information can be added by sliding the criteria along the slider. In Figure 3, the difference between "Capacity" and "Accessibility" is smaller than between "Accessibility" and "Cost". The overlap between the visual clouds for the former two indicate that the decision-maker is ambiguous with respect to the rank order of these two criteria, whereas he/she is confident in the rank order between the latter two. The gap between is due to that the minimum difference between them is greater than zero. When using a graphical user interface of this kind and distributing weights in this way the user can interactively adjust the distances between the criteria until the distances depict an adequate representation of the situation.

The decision-maker may redo the procedure if new information (or a better understanding) has been added. At no point of the procedure the decision-makers have to be precise. Thus, a cardinal ranking (in this sense) is consequently imposed over all the criteria. This requires $\mathrm{N}$ judgements. Thereafter, criteria weights can be adjusted if desired. Depending of the level of interaction the amount of adjustment might of course vary. Note that despite being the case in the current graphical user interface, the ranking does not have to be a total order.

\section{Discussion}

In prescriptive decision analysis, perceptions are subject to change and evolve over time, and the representation of these perceptions should be dynamic. Beliefs and preferences are not static, and the decision-maker's view on what is important (and the relations) for the

\footnotetext{
${ }^{\dagger}$ With no cardinal information the procedure produces ROC weights.
} 
decision may change during the progress of the decision process.

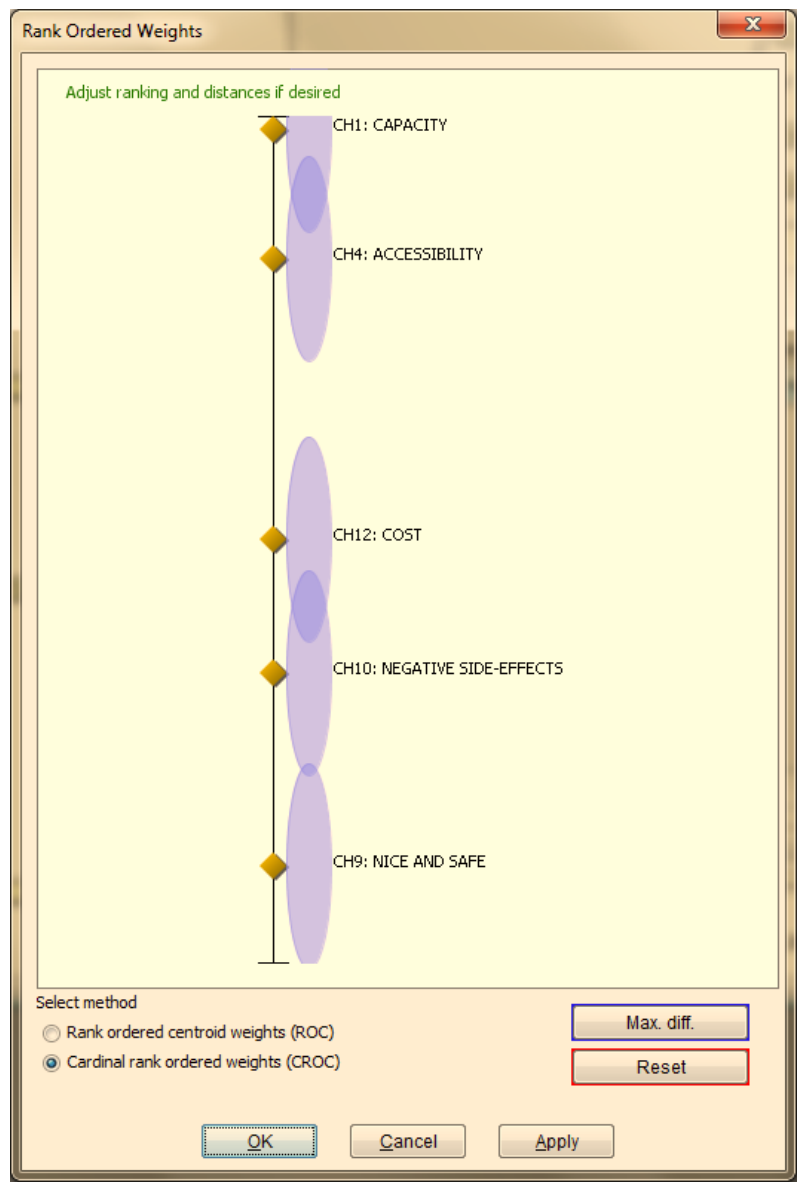

Fig 3. CROC weight elicitation after the cardinal rank ordering step

The CROC method was employed in three case studies. The decisions faced in the cases were of the same magnitude, influencing citizens in urban areas. Case 1 is described in more detail in Refs. 36-38, case 2 in Ref. 35, and case 3 in Ref. 39. Here, we compare the real-life elicitation aspects of the three cases.

In case 1 , the stakeholders asked for weight statements were the decision making politicians. The case focussed on evaluating seven competing environmental planning strategies in the municipality of Örebro having approximately 130,000 inhabitants. Prior to a first weighting round, two joint workshops were held in order to generate the set of fundamental evaluation criteria. Following this, four consecutive joint workshops were held involving the civil servants and politicians focussing on appraisal of the alternatives, includ- ing generation of means criteria and indicators for appraisal. After these workshops, a second weight elicitation round was performed, followed by two joint workshops for analysing and discussing decision evaluation results.

Case 2 treated the same decision problem as case 1 , but the stakeholders asked for weight statements were high school students in the municipality. A web application for gathering of larger amounts of stakeholder preferences was set up. Weight elicitation was conducted at two separate occasions and between these two occasions the students were partitioned into three groups, where group 1 did not discuss the issue, group 2 had teacher assisted discussions and group 3 had a discussion using a web forum.

In the cases $1-2$, both the weight elicitation methods direct weight assessment and CROC were employed twice at two different occasions a couple of months apart. The settings in the cases 1 and 2 were similar in terms of type of decision-problem and decision process, yet the elicitation outcome of these two cases differed a lot. Since the two elicitation procedures employed varied regarding the elicitation components (extraction, representation, and interpretation), it is useful to compare these aspects. In case 1, statements from both elicitation occasions were represented in constraint sets generated by the interpretation stage. When the changes are minor (which could be attributed to unintended change) the constraint sets are practically identical. In case 1 , nearly all changes were minor and resulted in identical interpretations. In case 2, employing direct weight assessment, the number of major changes (which could not be attributed to unintended change) were many more than what would be expected when relating them to the similar situation in case 1 . This can partly be attributed to the fact that minor (unintended) changes in statements on importance cannot be handled realistically in the interpretation of the direct method. The imprecision even in the ordinal weight information provided by the decision-makers suggests that there is a need for methods that can handle such input.

In case 3 , the CROC method was employed to weigh the criteria of "Environmental impact" assessed from life-cycle cost evaluations, "Ethics" assessed by scoring the alternatives under consideration against the UN millennium targets, and “Cost” in a decision regarding destruction of hazardous materials. The CROC method was used and the ranking of criteria was done in 
a SWING approach. Due to the highly numerically imprecise properties of the utility assessments as well as the scoring procedure of the alternatives, ratio based elicitation methods were deemed as less meaningful than rank based approaches. Still, it was possible to identify a most preferred alternative.

\section{Conclusion}

This paper sums up and explicates the theoretical and empirical work with the CROC method. The outcome of the comparison between the cases was that the CROC interpretation captures the imprecision and ambiguity of decision-maker preferences in a realistic manner and that the method was more robust and persistent to noisy input. Further, it involves less decision-maker judgments than interval-based methods, making it feasible when the number of criteria grows, as well as the fact that it can be employed without forcing a decisionmaker to make quantitative statements. The CROC method's extraction part is graphical and its design encourages imprecision, rendering it suitable for decision analysis processes where imprecision and ambiguity are prevailing both with respect to the performance of decision alternatives and the relative importance of criteria.

The software utilised in the article can be downloaded from www.preference.bz.

\section{Acknowledgements}

The research was funded by the Swedish research council FORMAS, grant 2011-3313-20412-31, and by strategic funds from the Swedish government within ICT The Next Generation.

\section{References}

1. R. Keeney and H. Raiffa, Decisions with Multiple Objectives: Preferences and Value Trade-offs (John Wiley, NY, 1976).

2. R. Keeney, Making better decision makers, in Decision Analysis 1(4) (2004) 193-204.

3. D. von Winterfeldt and W. Edwards, Decision Analysis and Behavioural Research (Cambridge University Press, NY, 1986).

4. V. Belton and T. Stewart, Multiple Criteria Decision Analysis: An Integrated Approach (Kluwer Academic Publishers, UK, 2002).

5. T. Saaty, How to make a decision: the analytic hierarchy process, in Interfaces 24(6) (1994) 19-43.
6. B. Roy, The outranking approach and the foundation of Electre methods, in Theory and Decision 31(1) (1991) 49-73.

7. J.P. Brans, Ph. Vincke and B. Mareschal, How to select and how to rank projects: the PROMETHEE method, in European Journal of Operational Research 24(2) (1986) 228-238.

8. R.V. Brown, Making decision research useful - not just rewarding, in Judgment and Decision Making 1(2) (2006) 162-173.

9. J. Wallenius, J.S. Dyer, P.C. Fishburn, R.E. Steuer, S. Zionts and K. Deb, Multiple criteria decision making, multiattribute utility theory: recent accomplishments and what lies ahead, in Management Science 54(7) (2008) 1336-1349.

10. S. French and D.-L. Xu, Comparison study of multiattribute decision analytic software, in Journal of MultiCriteria Decision Analysis 13(2-3) (2005) 65-80.

11. R.T. Clemen and T. Reilly, Making Hard Decisions with Decision Tools Suite (Duxburry Press, 1999).

12. F. Barron and B. Barrett, Decision quality using ranked attribute weights, in Management Science 42(11) (1996) 1515-1523.

13. Z. Shapira, Risk Taking: A Managerial Perspective (Russel Sage Foundation, NY, 1995).

14. J. Corner and P Corner, Characteristics of decisions in decision analysis practice, in Journal of the Operational Research Society 46(3) (1995) 304-314.

15. A. Tversky and D. Kahneman, Rational choice and the framing of decisions, in Journal of Business 59(4) (1986) 251-278.

16. S. Lichtenstein and P. Slovic (eds.), The Construction of Preference (Cambridge University Press, NY, 2006).

17. J. Pictet and D. Bollinger, Extended use of the cards procedure as a simple elicitation technique for MAVT. Application to public procurement in Switzerland, in European Journal of Operational Research 185(3) (2008) 1300-1307.

18. P. Bottomley and J. Doyle, A comparison of three weight elicitation methods: good, better, and best, in Omega 29(6) (2001) 553-560.

19. K. Katsikopoulos and B. Fasolo, New tools for decision analysts, in IEEE Transactions on Systems, Man and Cybernetics Part A: Systems and Humans 36(5), (2006) 960-967.

20. E.U. Choo, B. Schoner and W.C. Wedley, Interpretation of criteria weights in multicriteria decision making, in Computers \& Industrial Engineering 37(3) (1999) 527 541.

21. C.R. Fox and K.E. See, Belief and preference in decision under uncertainty, in Thinking: Psychological Perspectives on Reasoning, Judgement and Decision Making, eds. D. Hardman and L. Macchi (John Wiley \& Sons, 2003).

22. D. Ellsberg, Risk, ambiguity, and the Savage axioms, in Quarterly Journal of Economics 75(4) (1961) 643-669. 
23. A. Tversky and D. Kahneman, The framing of decisions and the psychology of choice, in Science 211(4481) (1981) 453-458.

24. L.A. Lenert and J.R. Treadwell, Effects on preferences of violations of procedural invariance, in Medical Decision Making 19(4) (1999) 473-481.

25. M. Pöyhönen and R.P. Hämäläinen, On the convergence of multiattribute weighting methods, in European Journal of Operational Research 129(3) (2001) 569-585.

26. A. Larsson, J. Johansson, L. Ekenberg and M. Danielson, Decision Analysis with Multiple Objectives in a Framework for Evaluating Imprecision, in International Journal of Uncertainty, Fuzziness, and Knowledge-Based Systems, 13(5) (2005) 495-509.

27. K.S. Park, Mathematical programming models for characterizing dominance and potential optimality when multicriteria alternative values and weights are simultaneously incomplete, in IEEE Transactions on Systems, Man and Cybernetics- Part A: Systems and Humans 34(5) (2004) 601-614.

28. A. Larsson, J. Johansson, L. Ekenberg and M. Danielson, Decision analysis with multiple objectives in a framework for evaluating imprecision", in International Journal of Uncertainty, Fuzziness and Knowledge-Based Systems 13(5) (2005) 495-509.

29. J. Jia, G.W. Fischer and J. Dyer, Attribute weighting methods and decision quality in the presence of response error: a simulation study, in Journal of Behavioral Decision Making 11(2) (1998) 85-105.

30. M. Danielson and L. Ekenberg, Computing upper and lower bounds in interval decision trees, in European Journal of Operational Research 181(2) (2007) 808-816.

31. A. Jiménez, S. Rios-Insua and A. Mateos, A generic multi-attribute analysis system", in Computers \& Operational Research 33(4) (2006) 1081-1101.

32. J. Mustajoki, R.P. Hämäläinen and A. Salo, Decision support by interval SMART/SWING - incorporating imprecision in the SMART and SWING methods, in Decision Sciences 36(2) (2005) 317-339.

33. W.G. Stillwell, D.A. Seaver and W. Edwards, A comparison of weight approximation techniques in multiattribute decision making, in Organizational Behavior and Human Performance 28(1) (1981) 62-77.

34. W. Edwards and F.H. Barron, SMARTS and SMARTER: Improved simple methods for multiattribute utility measurement, in Organizational Behavior and Human Decision Processes 60(3) (1994) 306-325.

35. M. Riabacke, M. Danielson, L. Ekenberg and A. Larsson, A Prescriptive Approach for Eliciting Imprecise Weight Statements in an MCDA Process, in Proceedings of the 1st International Conference on Algorithmic Decision Theory (2009) 168-179.

36. M. Danielson, L. Ekenberg, J. Idefeldt and A. Larsson, Using a Software Tool for Public Decision Analysis - the Case of Nacka Municipality, in Decision Analysis 4(2) (2007) 76-90.
37. M. Danielson, L. Ekenberg, A. Ekengren, T. Hökby, and J. Lidén, Decision Process Support for Participatory Democracy, in Journal of Multi-Criteria Decision Analysis, 15(1-2) (2008) 15-30.

38. M. Danielson, L. Ekenberg, A. Larsson and M. Riabacke, Transparent public decision making: Discussion and case study in Sweden, in E-Democracy: A Group Decision and Negotiation Perspective, (Rios Insua, D. and French, S., eds., Springer, 2010).

39. E. Ekener-Petersen, G. Finnveden, A. Larsson, P. Sandin and K. Alverbro, A framework tool for integrated decision making involving ethical issues - case study on destruction of ammunition, forthcoming. 